\title{
A Comparison of Point Target Spectra Derived for Bistatic SAR Processing
}

\author{
Yew Lam Neo, Frank H. Wong, and Ian G. Cumming, Life Senior Member, IEEE
}

\begin{abstract}
The existence of a double hyperbola in the bistatic range equation makes it difficult to find an exact analytical solution for the 2-D point target spectrum. Several approximate solutions for the spectrum have been derived and used to focus bistatic synthetic aperture radar data. In this paper, we establish the relationship between three independently derived bistatic point target spectra. The first spectrum is Loffeld's bistatic formula, which consists of a quasi-monostatic and a bistatic phase term. The second spectrum makes use of Rocca's smile operator, which transforms bistatic data in a defined configuration to a monostatic equivalent. The third spectrum is derived using a power series-called the method of series reversion (MSR). The MSR spectrum is the most general among the three. This paper shows that this spectrum can be reduced to the same formulation as the former two when certain conditions are met. In addition, a new approximate spectrum is derived using a Taylor series expansion about the two stationary phase points of the transmitter and receiver. We also give an alternative geometrical proof of the relationship between Rocca's smile operator and Loffeld's bistatic deformation term. The accuracies of the point target spectra are demonstrated using simulations of an $\mathrm{X}$-band bistatic airborne radar with a fixed baseline.
\end{abstract}

Index Terms-Bistatic synthetic aperture radar (SAR), Loffeld's bistatic formula (LBF), Rocca's smile, series reversion, 2-D spectrum.

\section{INTRODUCTION}

$\mathbf{T}$ HE FORMULATION of a point target spectrum is a key step in deriving synthetic aperture radar (SAR) focusing algorithms, which exploits the processing efficiency of the frequency domain [1]. The general bistatic SAR range equation has two square roots, referred to as the double-square-root (DSR) term. This DSR term makes it difficult to apply the method of stationary phase [2] directly in order to obtain an analytical solution for the 2-D spectrum.

A survey of the literature shows that there are three generic approaches to dealing with this inversion problem. The first method is to solve the problem by modeling the SAR transfer function numerically [3]-[6]. These methods tend to be more

Manuscript received December 14, 2006; revised October 15, 2007. The work of Y. L. Neo was supported by DSO National Laboratories (Singapore).

Y. L. Neo is with the Sensor Division, Radar Techniques Laboratory, DSO National Laboratories, Singapore 118230 (e-mail: nyewlam@dso.org.sg).

F. H. Wong is with MacDonald, Dettwiler and Associates Ltd., Richmond, BC V6V 2J3, Canada, and also with the Radar Remote Sensing Group, Department of Electrical and Computer Engineering, University of British Columbia, Vancouver, BC V6T 1Z4, Canada (e-mail: fhw@mda.ca).

I. G. Cumming is with the Department of Electrical and Computer Engineering, University of British Columbia, Vancouver, BC V6T 1Z4, Canada (e-mail: ianc@ece.ubc.ca).

Color versions of one or more of the figures in this paper are available online at http://ieeexplore.ieee.org.

Digital Object Identifier 10.1109/TGRS.2008.919018 computationally intensive than methods that use analytical models of the spectrum.

The second approach is to transform the bistatic data to a monostatic equivalent. In [7] and [8], a convolution operator called Rocca's smile is used, which is based upon the technique of dip move out (DMO) [9]. In [6], Bamler et al. investigate an equivalent velocity to model the bistatic configuration with a monostatic equivalent. However, any method that reduces to a monostatic configuration may not be accurate for extreme bistatic cases because the sum of the two square roots generally cannot be scaled into a single hyperbolic function, i.e., a single square root [3], [10], [11].

The third method is to solve for the 2-D spectrum directly by using the method of stationary phase. An approximate analytical solution for the general bistatic 2-D frequency spectrum has been proposed in [11]. Its relationship with the DMO method is presented in [12]. Notably, several algorithms have been formulated based on Loffeld's bistatic formula (LBF) [13], [14]. Recently, a power series method [15] for the general bistatic case has been formulated. The spectrum of a single point target is derived based on the method of series reversion (MSR) [16], which gives a more accurate formulation of the stationary point in the form of a power series. The accuracy of this method is "scalable" in the sense that its accuracy depends on the number of terms used in the power series. We refer to this method of deriving the bistatic spectrum as MSR.

This paper shows the relationships between three methods used to derive the 2-D bistatic spectrum: the LBF, DMO, and MSR methods. We begin with a short discussion of a general bistatic signal model in Section II, where we introduce common notations that are used for each of the signal spectra. Next, we give brief derivations and short descriptions of the MSR (Section II-B) and LBF (Section II-C) methods. Then, in Section III, we introduce a new method of deriving the spectrum, the two-stationary-phase-point (TSPP) method, and use it to describe algebraically how the LBF and MSR spectra relate to one another. We illustrate their relation using simulations in Section IV. In the Appendix, we give a geometric interpretation of the DMO method, and in Section V, we use a geometrical proof to define a relationship between the LBF and DMO methods. Simulations are performed in Section VI to illustrate the focusing accuracies of the LBF, DMO, and MSR forms of the spectrum.

\section{Two-Dimensional Bistatic Spectrum}

In this section, we briefly introduce a bistatic SAR signal model and summarize two methods of formulating the bistatic 


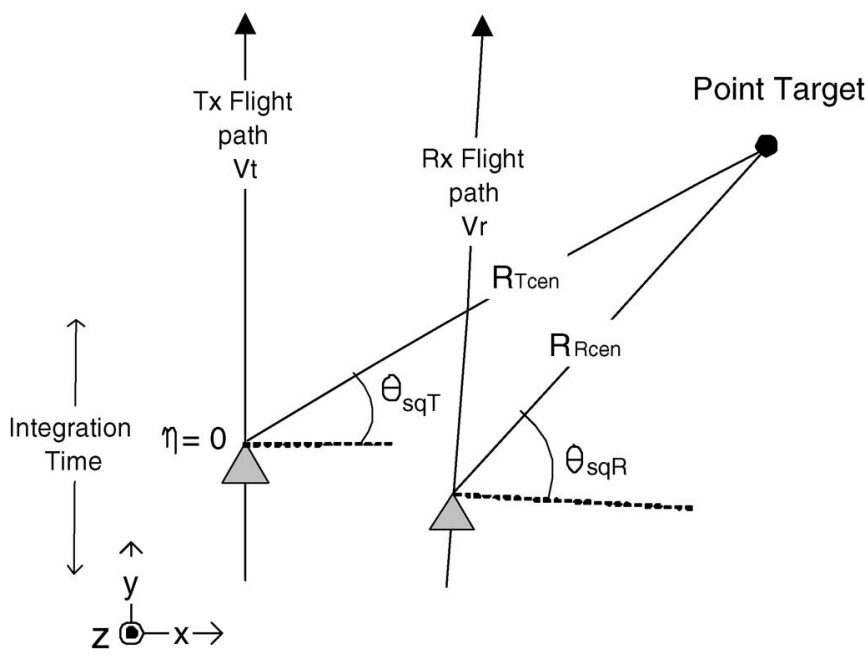

Fig. 1. Signal and image data in a bistatic configuration.

spectrum-the LBF of [11] and the MSR of [15]. The approaches of both formulations are similar: An analytical function in frequency is derived by using the method of stationary phase [2]. The accuracy of the spectrum differs in the two cases because of the different assumptions used.

\section{A. Bistatic SAR Signal Model}

The point target spectrum used in this paper is based on a 2-D spectrum of the reference point target derived in [15] and repeated here for convenience

$$
\begin{aligned}
& S\left(f_{\tau}, f_{\eta}\right) \approx W_{r}\left(f_{\tau}\right) W_{\text {az }}\left(f_{\eta}+\left(f_{o}+f_{\tau}\right) \frac{k_{1}}{c}\right) \\
& \times \exp \left\{-j\left[\phi_{\mathrm{T}}\left(\eta\left(f_{\eta}\right)\right)+\phi_{\mathrm{R}}\left(\eta\left(f_{\eta}\right)\right)\right]\right\}
\end{aligned}
$$

where $W_{r}$ represents the spectral shape of the transmitted pulse and $W_{\text {az }}$ represents the shape of the received Doppler spectrum. The parameter $k_{1}$ is the first derivative of the expanded range equation in azimuth time $\eta$, which is given in [15]. The variable $f_{\eta}$ is the azimuth frequency, $f_{\tau}$ is the range frequency, $f_{o}$ is the carrier frequency, and $c$ is the speed of light. The variable $\eta\left(f_{\eta}\right)$ contains the relationship between azimuth frequency $f_{\eta}$ and azimuth time $\eta$, which is found by solving for the stationary phase point [2].

The geometry of the bistatic SAR data collection is shown in Fig. $1 . R$ is the instantaneous range to the point target, and the subscripts " $T$ " and " $R$ " refer to the transmitter and receiver, respectively. The subscript "cen" refers to the geometry at time $\eta=0$, i.e., the "Doppler centroid" time when the target is in the center of the combined beam. At this time, the ranges to the target are $R_{\text {Tcen }}$ and $R_{\text {Rcen }}$, and the squint angles are $\theta_{\mathrm{sqT}}$ and $\theta_{\mathrm{sqR}}$.

The variable $\phi_{\mathrm{T}}$ is the transmitter-based phase term, and $\phi_{\mathrm{R}}$ is the receiver-based phase term in the spectrum [(1)], which are given by

$$
\begin{aligned}
& \phi_{\mathrm{T}}(\eta)=2 \pi \frac{\left(f_{o}+f_{\tau}\right)}{c} R_{\mathrm{T}}(\eta)+\pi f_{\eta} \eta \\
& \phi_{\mathrm{R}}(\eta)=2 \pi \frac{\left(f_{o}+f_{\tau}\right)}{c} R_{\mathrm{R}}(\eta)+\pi f_{\eta} \eta .
\end{aligned}
$$

Each of the two range functions $R_{\mathrm{T}}$ and $R_{\mathrm{R}}$ has a hyperbolic form, and the sum of the two functions gives rise to the DSR form of the bistatic range function $R=R_{\mathrm{T}}+R_{\mathrm{R}}$. For simplicity, we have dropped the $f_{\eta}$ dependence of $\eta$ in these and subsequent equations.

While an approximate analytical solution exists for $\eta\left(f_{\eta}\right)$ in the monostatic case [17], no simple analytical solution is available for this time-frequency relationship in the bistatic case. The problem lies in the inversion of the DSR. In the following two sections, we briefly examine how the MSR and LBF derivations of the bistatic spectra handle the inversion problem.

\section{B. Two-Dimensional Spectrum Derived Using the MSR}

The MSR expands the phase history around the beam center at zero azimuth time [15]. By expanding the instantaneous bistatic range $R(\eta)$ in azimuth time as a Taylor series around $\eta=0$ and solving for the bistatic stationary phase point by using the series reversion formula [15], [16], a power series representation of $\eta\left(f_{\eta}\right)$ (which we denote by $\left.\widetilde{\eta}_{b}\right)$ is obtained

$$
\begin{aligned}
\widetilde{\eta}_{b}=A_{1}\left(-\frac{c f_{\eta}}{f_{o}+f_{\tau}}-\right. & \left.k_{1}\right)+A_{2}\left(-\frac{c f_{\eta}}{f_{o}+f_{\tau}}-k_{1}\right)^{2} \\
& +A_{3}\left(-\frac{c f_{\eta}}{f_{o}+f_{\tau}}-k_{1}\right)^{3}+\cdots
\end{aligned}
$$

and the coefficients are given by

$$
\begin{aligned}
A_{1} & =\frac{1}{2 k_{2}} \\
A_{2} & =-\frac{3 k_{3}}{8 k_{2}^{3}} \\
A_{3} & =\frac{9 k_{3}^{2}-4 k_{2} k_{4}}{16 k_{2}^{5}} \\
\ldots &
\end{aligned}
$$

where the derivatives $k_{2}, k_{3}$, and $k_{4}$ of the expanded version of the range equation in azimuth time $\eta$ are given in [15] and

$$
\begin{array}{r}
k_{\mathrm{N}}=k_{\mathrm{TN}}+k_{\mathrm{RN}}=\left.\left\{\frac{d^{N} R_{\mathrm{T}}(\eta)}{d \eta^{N}}+\frac{d^{N} R_{\mathrm{R}}(\eta)}{d \eta^{N}}\right\}\right|_{\eta=0}, \\
\text { where } N \in Z^{+}
\end{array}
$$

Substituting $\widetilde{\eta}_{b}$ for $\eta\left(f_{\eta}\right)$ in (1), the bistatic spectrum derived using the MSR can be written as

$$
\begin{aligned}
S\left(f_{\tau}, f_{\eta}\right) \approx W_{r}\left(f_{\tau}\right) W_{\mathrm{az}} & \left(f_{\eta}+\left(f_{o}+f_{\tau}\right) \frac{k_{1}}{c}\right) \\
& \times \exp \left\{-j\left[\phi_{\mathrm{T}}\left(\widetilde{\eta}_{b}\right)+\phi_{\mathrm{R}}\left(\widetilde{\eta}_{b}\right)\right]\right\} .
\end{aligned}
$$

The accuracy of this spectrum is affected by the number of terms used in the expansion [(4)]. This is a key feature of the MSR formulation. For a higher resolution, a wider aperture, or a larger bistatic angle, more phase terms need to be retained to achieve a specified level of accuracy in the spectrum [15]. 


\section{C. $L B F$}

Loffeld et al. [11] derived an approximate analytical solution for the point target response in the 2-D frequency domain by applying a Taylor series expansion to the one-way phase terms $\phi_{\mathrm{T}}(\eta)$ and $\phi_{\mathrm{R}}(\eta)$ in (2) and (3), respectively. The monostatic phase functions are expanded about their individual monostatic stationary phase points up to the second-order phase term, which allows the bistatic point target spectrum to be approximated by

$$
\begin{aligned}
S\left(f_{\tau}, f_{\eta}\right) \approx & W_{r}\left(f_{\tau}\right) W_{\mathrm{az}}\left(f_{\eta}+\left(f_{o}+f_{\tau}\right) \frac{k_{1}}{c}\right) \\
& \times \exp \left\{-j\left[\phi_{\mathrm{T}}\left(\widetilde{\eta}_{\mathrm{T}}\right)+\phi_{\mathrm{R}}\left(\widetilde{\eta}_{\mathrm{R}}\right)\right]\right\} \\
& \times \int \exp \left\{-j\left[\dot{\phi_{\mathrm{T}}}\left(\widetilde{\eta}_{\mathrm{T}}\right)\left(\eta-\widetilde{\eta}_{\mathrm{T}}\right)\right.\right. \\
& \left.\left.+\dot{\phi}_{\mathrm{R}}\left(\widetilde{\eta}_{\mathrm{R}}\right)\left(\eta-\widetilde{\eta}_{\mathrm{R}}\right)\right]\right\} d \eta \\
& \times \int \exp \left\{-\frac{j}{2}\left[\ddot{\phi}_{\mathrm{T}}\left(\widetilde{\eta}_{\mathrm{T}}\right)\left(\eta-\widetilde{\eta}_{\mathrm{T}}\right)^{2}\right.\right. \\
& \left.\left.+\ddot{\phi}_{\mathrm{R}}\left(\widetilde{\eta}_{\mathrm{R}}\right)\left(\eta-\widetilde{\eta}_{\mathrm{R}}\right)^{2}\right]\right\} d \eta
\end{aligned}
$$

where $\widetilde{\eta}_{\mathrm{T}}$ and $\widetilde{\eta}_{\mathrm{R}}$ are the solutions to the individual stationary phases of the transmitter and receiver phase histories, respectively. The terms $\dot{\phi}_{\mathrm{T}}\left(\widetilde{\eta}_{\mathrm{T}}\right)$ and $\dot{\phi_{\mathrm{R}}}\left(\widetilde{\eta}_{\mathrm{R}}\right)$ are both zero, as $\widetilde{\eta}_{\mathrm{T}}$ and $\widetilde{\eta}_{\mathrm{R}}$ are the stationary points of the transmitter and receiver phase histories, respectively. Thus, the first integral in (8) vanishes.

Equation (8) can be simplified by using the fact that the sum of two quadratic functions is a shifted and scaled quadratic function

$$
\begin{aligned}
a(x-b)^{2} & +c(x-d)^{2} \\
& =(a+c)\left[x-\frac{a b+c d}{a+c}\right]^{2}+\left[\frac{a c}{a+c}(b-d)^{2}\right] .
\end{aligned}
$$

Applying this fact and using the substitutions $a=\ddot{\phi}_{\mathrm{T}}\left(\widetilde{\eta}_{\mathrm{T}}\right), c=$ $\ddot{\phi}_{\mathrm{R}}\left(\widetilde{\eta}_{\mathrm{R}}\right), x=\eta, b=\widetilde{\eta}_{\mathrm{T}}$, and $d=\widetilde{\eta}_{\mathrm{R}}$, the last integral in (8) can be shown to be

$$
\begin{aligned}
I\left(f_{\eta}\right)= & \int \exp \left\{-\frac{j}{2}\left\{\frac{\ddot{\phi}_{\mathrm{T}}\left(\widetilde{\eta}_{\mathrm{T}}\right) \ddot{\phi}_{\mathrm{R}}\left(\widetilde{\eta}_{\mathrm{R}}\right)}{\ddot{\phi}_{\mathrm{T}}\left(\widetilde{\eta}_{\mathrm{T}}\right)+\ddot{\phi}_{\mathrm{R}}\left(\widetilde{\eta}_{\mathrm{R}}\right)}\left(\widetilde{\eta}_{\mathrm{T}}-\widetilde{\eta}_{\mathrm{R}}\right)^{2}\right.\right. \\
& \left.\left.+\left[\ddot{\phi}_{\mathrm{T}}\left(\widetilde{\eta}_{\mathrm{T}}\right)+\ddot{\phi}_{\mathrm{R}}\left(\widetilde{\eta}_{\mathrm{R}}\right)\right] \times\left(\eta-\widehat{\eta}_{b}\right)^{2}\right\}\right\} d \eta
\end{aligned}
$$

where

$$
\widehat{\eta}_{b}=\frac{\ddot{\phi}_{\mathrm{T}}\left(\widetilde{\eta}_{\mathrm{T}}\right) \widetilde{\eta}_{\mathrm{T}}+\ddot{\phi}_{\mathrm{R}}\left(\widetilde{\eta}_{\mathrm{R}}\right) \widetilde{\eta}_{\mathrm{R}}}{\ddot{\phi}_{\mathrm{T}}\left(\widetilde{\eta}_{\mathrm{T}}\right)+\ddot{\phi}_{\mathrm{R}}\left(\widetilde{\eta}_{\mathrm{R}}\right)} .
$$

Applying the method of stationary phase to (10), the approximate stationary phase point is given by $\widehat{\eta}_{b}$, and the spectrum is given by (ignoring any amplitude variations)

$$
\begin{aligned}
S_{2}\left(f_{\tau}, f_{\eta}\right) \approx & W_{r}\left(f_{\tau}\right) W_{\mathrm{az}}\left(f_{\eta}+\left(f_{o}+f_{\tau}\right) \frac{k_{1}}{c}\right) \\
& \times \exp \left\{-j \Psi_{1}\left(f_{\tau}, f_{\eta}\right)\right\} \\
& \times \exp \left\{-\frac{j}{2} \Psi_{2}\left(f_{\tau}, f_{\eta}\right)\right\}
\end{aligned}
$$

where

$$
\begin{aligned}
& \Psi_{1}\left(f_{\tau}, f_{\eta}\right)=\phi_{\mathrm{T}}\left(\widetilde{\eta}_{\mathrm{T}}\right)+\phi_{\mathrm{R}}\left(\widetilde{\eta}_{\mathrm{R}}\right) \\
& \Psi_{2}\left(f_{\tau}, f_{\eta}\right)=\frac{\ddot{\phi}_{\mathrm{T}}\left(\widetilde{\eta}_{\mathrm{T}}\right) \ddot{\phi}_{\mathrm{R}}\left(\widetilde{\eta}_{\mathrm{R}}\right)}{\ddot{\phi}_{\mathrm{T}}\left(\widetilde{\eta}_{\mathrm{T}}\right)+\ddot{\phi}_{\mathrm{R}}\left(\widetilde{\eta}_{\mathrm{R}}\right)}\left(\widetilde{\eta}_{\mathrm{T}}-\widetilde{\eta}_{\mathrm{R}}\right)^{2} .
\end{aligned}
$$

The first exponential term $\Psi_{1}$ is known as the quasi-monostatic term, whereas the second exponential term $\Psi_{2}$ is known as the bistatic deformation term. Unlike the MSR formulation of Section II-B, the accuracy of this spectrum is restricted by the limited number of terms used in (8) (it is a second-order expansion).

\section{Link BETwEEN THE LBF AND MSR SPECTRA}

In this section, we show that the spectrum derived using the MSR in Section II-B is a generalization of the LBF of Section II-C.

\section{A. Analytical Development}

Beginning with the bistatic phase term in (7), if we do Taylor series expansions of the phase term $\phi_{\mathrm{T}}\left(\widetilde{\eta}_{b}\right)$ about $\widetilde{\eta}_{\mathrm{T}}$ and the phase term $\phi_{\mathrm{R}}\left(\widetilde{\eta}_{b}\right)$ about $\widetilde{\eta}_{\mathrm{R}}$ [where $\widetilde{\eta}_{b}$ is the bistatic stationary point defined in (4)], the phase of the MSR formulation in (7) becomes

$$
\begin{aligned}
\phi_{\mathrm{T}}\left(\widetilde{\eta}_{b}\right)+\phi_{\mathrm{R}}\left(\widetilde{\eta}_{b}\right)= & \phi_{\mathrm{T}}\left(\widetilde{\eta}_{\mathrm{T}}+\Delta \widetilde{\eta}_{\mathrm{T}}\right)+\phi_{\mathrm{R}}\left(\widetilde{\eta}_{\mathrm{R}}+\Delta \widetilde{\eta}_{\mathrm{R}}\right) \\
= & \phi_{\mathrm{T}}\left(\widetilde{\eta}_{\mathrm{T}}\right)+\phi_{\mathrm{R}}\left(\widetilde{\eta}_{\mathrm{R}}\right) \\
& +\frac{1}{2}\left(\Delta \widetilde{\eta}_{\mathrm{T}}^{2} \ddot{\phi}_{\mathrm{T}}\left(\widetilde{\eta}_{\mathrm{T}}\right)+\Delta \widetilde{\eta}_{\mathrm{R}}^{2} \ddot{\phi}_{\mathrm{R}}\left(\widetilde{\eta}_{\mathrm{R}}\right)\right) \\
& \left.+\frac{1}{3 !}\left(\Delta \widetilde{\eta}_{\mathrm{T}}^{3} \ddot{\phi}_{\mathrm{T}}\right)+\Delta \widetilde{\eta}_{\mathrm{R}}^{3} \ddot{\phi}_{\mathrm{R}}\left(\widetilde{\eta}_{\mathrm{R}}\right)\right) \\
& \ldots
\end{aligned}
$$

where

$$
\begin{aligned}
\Delta \widetilde{\eta}_{\mathrm{T}} & =\widetilde{\eta}_{b}-\widetilde{\eta}_{\mathrm{T}} \\
\Delta \widetilde{\eta}_{\mathrm{R}} & =\widetilde{\eta}_{b}-\widetilde{\eta}_{\mathrm{R}} .
\end{aligned}
$$

The terms on the right-hand side of (16) and (17) are the azimuth times measured from the respective stationary phase points of the transmitter and receiver. Note that $\dot{\phi}_{\mathrm{R}}\left(\widetilde{\eta}_{\mathrm{R}}\right)$ and $\dot{\phi}_{\mathrm{T}}\left(\widetilde{\eta}_{\mathrm{T}}\right)$ are both zero because of the definition of the stationary points. As a result, they do not appear in (15).

The phases on the left-hand side of (15) represent the MSR in (7). The expansion on the right-hand side of (15) is the formulation leading to the link with the LBF. This formulation is new, and we refer to it as the TSPP method.

The TSPP formulation of the bistatic spectrum has a pair of quasi-monostatic phase terms that are the same as the quasimonostatic phase terms in LBF (14). If we approximate ${ }^{1} \widetilde{\eta}_{b}$ by

\footnotetext{
${ }^{1}$ Note that $\widetilde{\eta}_{b}$ is the stationary point or the azimuth time/frequency relationship using MSR. $\widehat{\eta}_{b}$ is a mathematical approximation of $\widetilde{\eta}_{b}$ derived using LBF.
} 
$\widehat{\eta}_{b}$ and consider only the quadratic terms in (15), the sum of the quadratic phase terms becomes

$$
\begin{aligned}
& \frac{1}{2}\left[\Delta \widetilde{\eta}_{\mathrm{T}}^{2} \ddot{\phi}_{\mathrm{T}}\left(\widetilde{\eta}_{\mathrm{T}}\right)+\Delta \widetilde{\eta}_{\mathrm{R}}^{2} \ddot{\phi}_{\mathrm{R}}\left(\widetilde{\eta}_{\mathrm{R}}\right)\right] \\
& \quad \approx \frac{1}{2}\left[\left(\widehat{\eta}_{b}-\widetilde{\eta}_{\mathrm{T}}\right)^{2} \ddot{\phi}_{\mathrm{T}}\left(\widetilde{\eta}_{\mathrm{T}}\right)+\left(\widehat{\eta}_{b}-\widetilde{\eta}_{\mathrm{R}}\right)^{2} \ddot{\phi}_{\mathrm{R}}\left(\widetilde{\eta}_{\mathrm{R}}\right)\right] .
\end{aligned}
$$

Using the results in (9), the sum of these quadratic phase terms is equivalent to the bistatic deformation term in (12) when the condition $\widetilde{\eta}_{b} \approx \widehat{\eta}_{b}$ holds, i.e.,

$$
\begin{aligned}
\frac{1}{2}\left[\left(\widehat{\eta}_{b}-\widetilde{\eta}_{\mathrm{T}}\right)^{2} \ddot{\phi}_{\mathrm{T}}\left(\widetilde{\eta}_{\mathrm{T}}\right)+\left(\widehat{\eta}_{b}-\widetilde{\eta}_{\mathrm{R}}\right)^{2} \ddot{\phi}_{\mathrm{R}}\left(\widetilde{\eta}_{\mathrm{R}}\right)\right] \\
\approx \frac{1}{2} \frac{\ddot{\phi}_{\mathrm{T}}\left(\widetilde{\eta}_{\mathrm{T}}\right) \ddot{\phi}_{\mathrm{R}}\left(\widetilde{\eta}_{\mathrm{R}}\right)}{\ddot{\phi}_{\mathrm{T}}\left(\widetilde{\eta}_{\mathrm{T}}\right)+\ddot{\phi}_{\mathrm{R}}\left(\widetilde{\eta}_{\mathrm{R}}\right)}\left(\widetilde{\eta}_{\mathrm{T}}-\widetilde{\eta}_{\mathrm{R}}\right)^{2} .
\end{aligned}
$$

The expression on the right-hand side of (19) is equal to $\Psi_{2} / 2$ of (14). Thus, the LBF is shown to be a special case of the point target spectrum formulation of the MSR given in (15).

\section{B. Observations on Bistatic Configurations}

The LBF method is accurate under two conditions. The first condition is that the higher order phase terms of (15) must be negligible because there is a truncation of the azimuth phase power series before applying the method of stationary phase. This limitation has been discussed in [11] and becomes significant at wider aperture, longer wavelength, and/or extreme bistatic cases.

Another necessary condition for the LBF to be valid is for $\widetilde{\eta}_{b} \approx \widehat{\eta}_{b}$. This condition determines the type of bistatic configurations that the LBF is able to focus. Due to the complexity of (4) and (11) and the wide range of configurations available for bistatic platforms, it is difficult to determine these bistatic configurations analytically.

Instead of considering the validity of the approximation over all azimuth frequencies, we can simplify the analysis by just considering the mean azimuth frequency (the Doppler centroid). That is, we assume that $\widetilde{\eta}_{b}\left(f_{\eta_{c}}\right) \approx \widehat{\eta}_{b}\left(f_{\eta_{c}}\right)$, where $f_{\eta_{c}}$ is the mean azimuth frequency

$$
f_{\eta_{c}}=-\frac{f_{o}+f_{\tau}}{c} k_{1}
$$

Substituting $f_{\eta_{c}}$ for $f_{\eta}$ in (4) causes all the terms in the brackets to become zero, and the mean value of the bistatic stationary point $\widetilde{\eta}_{b}\left(f_{\eta_{c}}\right)$ also becomes zero. Thus, from (11), and assuming that $\widetilde{\eta}_{b}\left(f_{\eta_{c}}\right) \approx \widehat{\eta}_{b}\left(f_{\eta_{c}}\right)$, we have

$$
\left.\left[\ddot{\phi}_{\mathrm{T}}\left(\widetilde{\eta}_{\mathrm{T}}\right) \widetilde{\eta}_{\mathrm{T}}+\ddot{\phi}_{\mathrm{R}}\left(\widetilde{\eta}_{\mathrm{R}}\right) \widetilde{\eta}_{\mathrm{R}}\right]\right|_{f_{\eta}=f_{\eta_{c}}} \approx 0 .
$$

A power series representation of the stationary point $\widetilde{\eta}_{\mathrm{T}}$ can be derived from (4) by setting the receiver-based derivatives $k_{\mathrm{RN}}$ 's to be equal to the transmitter-based derivatives $k_{\mathrm{TN}}$ 's in (6), giving

$$
\widetilde{\eta}_{\mathrm{T}}=B_{1}\left(-\frac{c f_{\eta}}{f_{o}+f_{\tau}}-2 k_{\mathrm{T} 1}\right)+B_{2}\left(-\frac{c f_{\eta}}{f_{o}+f_{\tau}}-2 k_{\mathrm{T} 1}\right)^{2}+\cdots
$$

where the coefficients are given by

$$
\begin{gathered}
B_{1}=\frac{1}{4 k_{\mathrm{T} 2}} \\
B_{2}=-\frac{3 k_{\mathrm{T} 3}}{32 k_{\mathrm{T} 2}^{3}} \\
B_{3}=\frac{9 k_{\mathrm{T} 3}^{2}-4 k_{\mathrm{T} 2} k_{\mathrm{T} 4}}{128 k_{\mathrm{T} 2}^{5}} \\
\cdots .
\end{gathered}
$$

A similar expression for $\widetilde{\eta}_{\mathrm{R}}$ can be derived from (4) by setting the transmitter-based derivatives $k_{\mathrm{TN}}$ 's to be equal to the receiver-based derivatives $k_{\mathrm{RN}}$ 's in (6).

Substituting this pair of stationary points into (21) and considering only the first two terms in the power series, it can be shown that the condition $\widetilde{\eta}_{b}\left(f_{\eta_{c}}\right) \approx \widehat{\eta}_{b}\left(f_{\eta_{c}}\right)$ simplifies to

$$
\left(\frac{k_{\mathrm{T} 3}}{k_{\mathrm{T} 2}^{2}}+\frac{k_{\mathrm{R} 3}}{k_{\mathrm{R} 2}^{2}}\right)\left(k_{\mathrm{R} 1}-k_{\mathrm{T} 1}\right)^{2} \approx 0 .
$$

Using condition (24), the bistatic configurations where the LBF would work well can be determined. This condition is satisfied when the value inside either bracket is approximately zero.

Consider the case where the value of the second bracket in (24) is zero. A trivial case that satisfies this condition is the monostatic configuration where $k_{\mathrm{R} 1}=k_{\mathrm{T} 1}$. Bistatic cases that have a short baseline relative to the slant ranges would also fall into this category because $k_{\mathrm{R} 1} \approx k_{\mathrm{T} 1}$. This condition is also satisfied when $k_{\mathrm{R} 1} \approx 0$ and $k_{\mathrm{T} 1} \approx 0$, i.e., when both antennas are pointing roughly at broadside.

The value in the first bracket is approximately zero when the platforms are flying with the same velocity along the same flight path (the leader-follower or tandem configuration) and with the squint angle of the transmitter approximately the negative of the squint angle of the receiver $\left(\theta_{\mathrm{sqT}} \approx-\theta_{\mathrm{sqR}}\right)$. In such a case, the condition is satisfied as $k_{\mathrm{R} 3} \approx-k_{\mathrm{T} 3}$ and $k_{\mathrm{R} 2} \approx k_{\mathrm{T} 2}$, and the LBF results are accurate (see results in Section VI-B).

It should be noted that (24) does not show all possible bistatic configurations where LBF is applicable. However, it is a good guide to determine some possible configurations where LBF is valid.

\section{Simulations-PART 1 (LBF, TSPP, AND MSR)}

In this section, we simulate three equal-velocity paralleltrack stationary cases, with both platforms operating in stripmap mode, to compare and verify the accuracy of the point target spectra between the LBF, TSPP, and MSR methods. 
TABLE I

SiMULATION PARAMETERS FOR PART 1

\begin{tabular}{|c|c|c|}
\hline Simulation parameters & Transmitter & Receiver \\
\hline Platform velocity & $98 \mathrm{~m} / \mathrm{sec}$ & $98 \mathrm{~m} / \mathrm{sec}$ \\
\hline Center frequency & \multicolumn{2}{|c|}{$10.17 \mathrm{GHz}$} \\
\hline Range bandwidth & \multicolumn{2}{|c|}{$50 \mathrm{MHz}$} \\
\hline Doppler bandwidth & \multicolumn{2}{|c|}{$660 \mathrm{~Hz}$} \\
\hline Altitude & $1000 \mathrm{~m}$ & $1000 \mathrm{~m}$ \\
\hline Distance between airplanes & \multicolumn{2}{|c|}{$2000 \mathrm{~m}$} \\
\hline \multicolumn{3}{|c|}{ Case I } \\
\hline Integration time & \multicolumn{2}{|c|}{$2.63 \mathrm{sec}$} \\
\hline Range to point target & $3751 \mathrm{~m}$ & $1915 \mathrm{~m}$ \\
\hline Squint angle & $5^{\circ}$ & $9.83^{\circ}$ \\
\hline \multicolumn{3}{|c|}{ Case II } \\
\hline Integration time & \multicolumn{2}{|c|}{$2.89 \mathrm{sec}$} \\
\hline Range to point target & $3794 \mathrm{~m}$ & $1999 \mathrm{~m}$ \\
\hline Squint angle & $10^{\circ}$ & $19.25^{\circ}$ \\
\hline \multicolumn{3}{|c|}{ Case III } \\
\hline Integration time & \multicolumn{2}{|c|}{$4.01 \mathrm{sec}$} \\
\hline Range to point target & $3976 \mathrm{~m}$ & $2326 \mathrm{~m}$ \\
\hline Squint angle & $20^{\circ}$ & $35.78^{\circ}$ \\
\hline
\end{tabular}

\section{A. Simulation Parameters}

In each case, a single point target is simulated using the airborne SAR parameters given in Table I. The three cases differ in the squint angles simulated.

\section{B. Simulation Results}

The results of the simulations are shown in Figs. 2-4. Rectangular weighting is used for both azimuth and range processing to simplify the interpretation of results. The ideal impulse response width (IRW) is 1.06 samples in both range and azimuth. The ideal peak sidelobe ratio (PSLR) is $-13.3 \mathrm{~dB}$, and the ideal integrated sidelobe ratio (ISLR) is $-10.0 \mathrm{~dB}$.

Cases of low, moderate, and high squints are discussed in the next three sections. The range responses are not shown, as the point targets are well focused in range for all the cases. The range IRWs differ from the theoretical values by less than $1 \%$, and the PSLR and ISLR differ from the theoretical values by less than $1 \mathrm{~dB}$.

1) Case I: Low Squint ( $\left.5^{\circ}\right)$ : This first simulation is a bistatic formation, with both antennas pointing near broadside. Fig. 2(a) shows the point target focused using the LBF. Fig. 2(b) shows the same point target focused using the TSPP spectrum given in (15), which is expanded up to the quadratic term. The linear phase terms $k_{\mathrm{T} 1}$ and $k_{\mathrm{R} 1}$ are small in such a case; therefore, the condition in (24) holds, and $\widetilde{\eta}_{b} \approx \widehat{\eta}_{b}$. Thus, the focusing results in Fig. 2(a) and (b) do not differ significantly.

Fig. 2(c) shows the results, with the TSPP spectrum being expanded up to the cubic term, showing a small improvement over Fig. 2(b).

2) Case II: Moderate Squint $\left(10^{\circ}\right)$ : In the second simulation, both antennas are squinted to a point where conditions (24) and $\widetilde{\eta}_{b} \approx \widehat{\eta}_{b}$ no longer hold. The point target focused using the LBF in Fig. 3(a) is poorly focused. Fig. 3(b) shows the same point target focused using the TSPP spectrum given in (15), which is expanded up to the quadratic term. The TSPP focusing is much better than the LBF, with only a small degradation over the $5^{\circ}$ squint TSPP case of Fig. 2(b). An improved result can be obtained by including the cubic phase term in the TSPP expansion, as shown in Fig. 3(c).

3) Case III: High Squint $\left(20^{\circ}\right)$ : Finally, for cases with a more extreme bistatic configuration, ${ }^{2}$ there is a large difference in the location of the stationary phase points between $\widetilde{\eta}_{b}$ and each of $\widetilde{\eta}_{\mathrm{T}}$ and $\widetilde{\eta}_{\mathrm{R}}$, and the LBF cannot focus the data. Also, more terms would be required in the TSPP approach of (15) to focus the point target, making such an approach inefficient. Fig. 4(a) shows that, even with a TSPP expansion up to the sixth-order term, the target is still poorly focused. However, the point target can be focused by expanding $\widetilde{\eta}_{b}$ in (4) to the thirdorder term and then processing the spectrum derived using the MSR of (7). Proper focusing is achieved, as shown in Fig. 4(b).

\section{Discussion}

We introduced the TSPP method in Section III-A to show the relation between the methods of deriving the LBF and MSR spectra. However, the results of this section show why we do not recommend the use of the TSPP in the general bistatic case. Instead, we recommend that the MSR be used for more extreme bistatic configurations.

\section{Bistatic Deformation Term and Rocca's Smile}

The existence of the quasi-monostatic and bistatic phase terms in (12) and (15) suggests a two-step focusing approach for the LBF: the removal of the bistatic deformation followed by the application of a quasi-monostatic focusing step [11]. Such a two-step method is similar to the DMO algorithm put forward by D'Aria et al. [7], which uses Rocca's smile operator. In this section, a geometrical proof is given to show how the LBF bistatic deformation term, $\Psi_{2}$ in (14), is linked to Rocca's smile operator for the "constant offset" case of the tandem configuration (a stationary case).

\section{A. Link Between LBF and Rocca's Smile Operator}

A geometrical method [7], borrowed from seismic reflection surveying [9], is used to transform a bistatic configuration to a monostatic one. The bistatic platforms are restricted to traveling along the same path with equal and constant velocities, as shown in Fig. 5.

For this tandem case, Rocca's smile operator transforms the bistatic data to a monostatic equivalent, which corresponds to a single platform located at the midpoint of the two bistatic platforms. To do this transformation, range shift and phase

\footnotetext{
${ }^{2}$ Some authors use the term "high bistatic degree" to refer to extreme bistatic configurations. In our context, the bistatic degree refers to the magnitude of the difference between the location of the two stationary phase points. This parameter determines the complexity of the bistatic case in our analysis.
} 
(a)

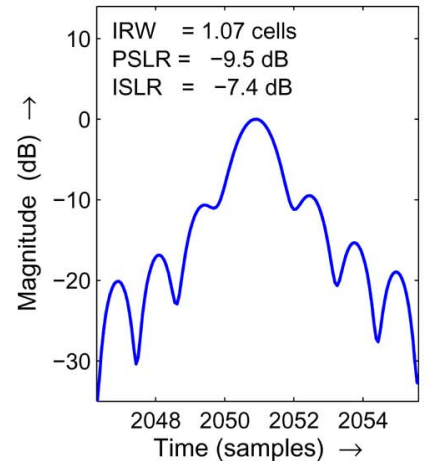

(b)

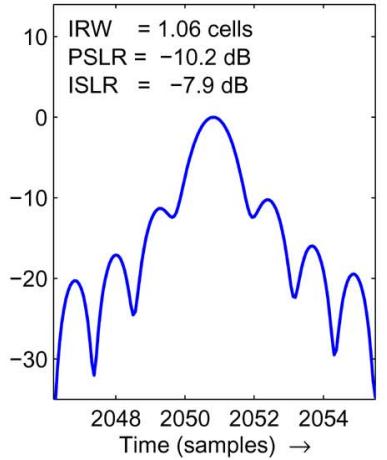

(c)

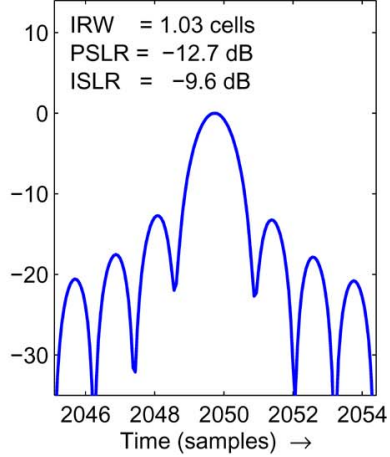

Fig. 2. Azimuth impulse responses for the LBF and two TSPP forms. (a) LBF. (b) TSPP (quadratic). (c) TSPP (cubic).

(a)

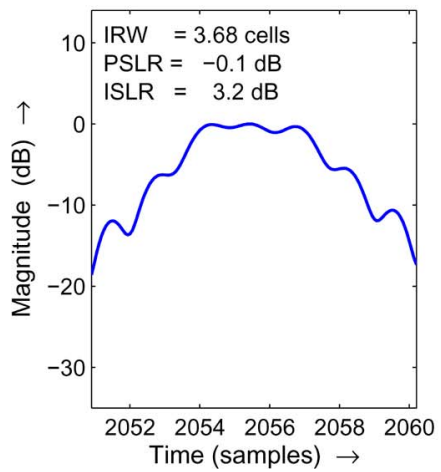

(b)

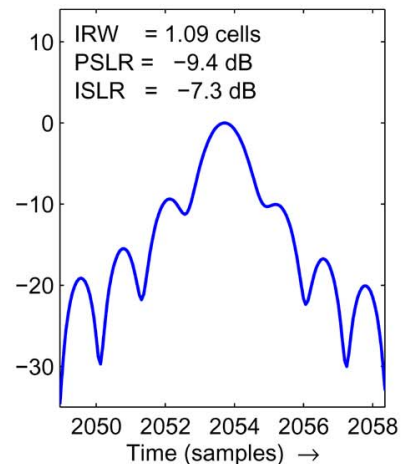

(c)

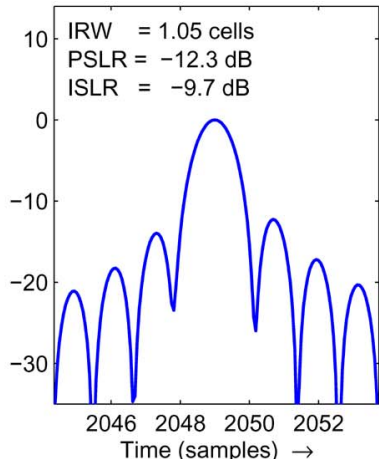

Fig. 3. Azimuth impulse responses for the LBF and two TSPP forms. (a) LBF. (b) TSPP (quadratic). (c) TSPP (cubic).

(a)

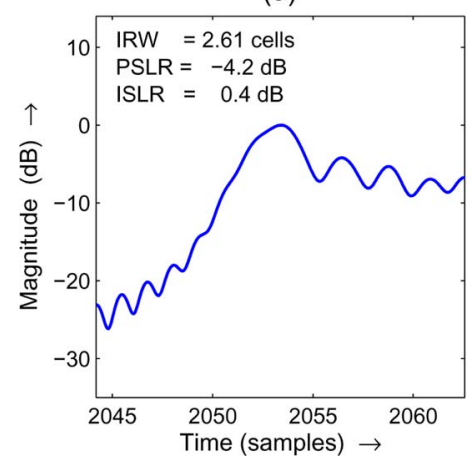

(b)

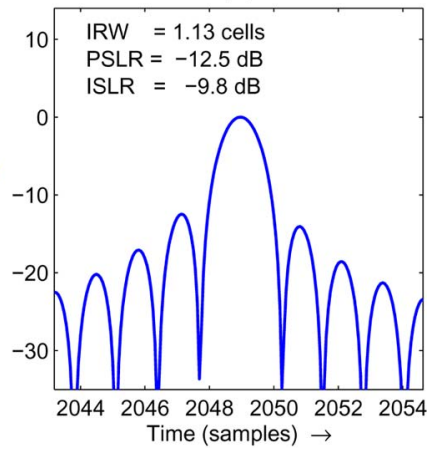

Fig. 4. Azimuth impulse responses. (a) Using the TSPP (up to the sixth-order term). (b) Using the MSR.

compensation are required-the shift corresponds to the travel time difference between the two geometries, which is given by

$$
t_{\mathrm{DMO}}\left(\theta_{\mathrm{sq}}\right)=t_{b}\left(\theta_{\mathrm{sq}}\right)-t_{m}\left(\theta_{\mathrm{sq}}\right)
$$

where $t_{b}$ is the bistatic round-trip travel time from the transmitter to the point target back to the receiver and $t_{m}$ is the roundtrip travel time between the equivalent monostatic antenna and the point target. The bistatic range to an arbitrary point is always greater than the two-way monostatic range to the same point, as shown in Fig. 6.

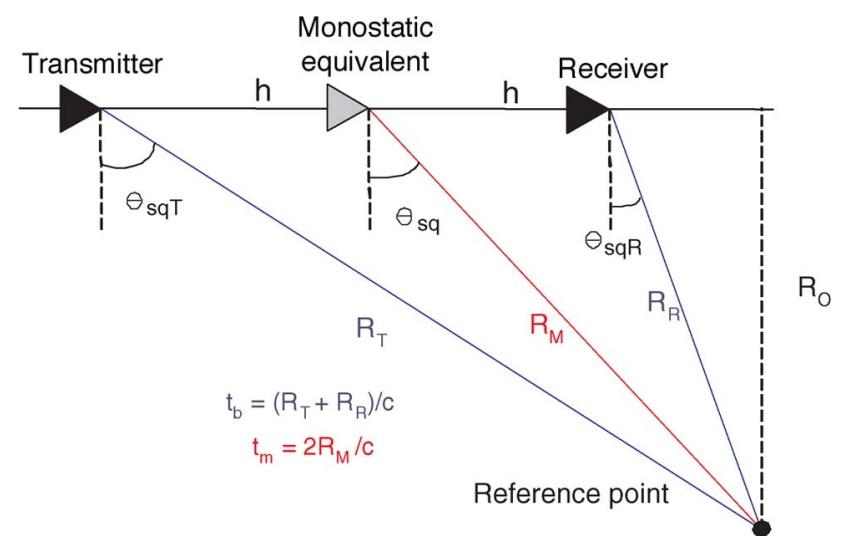

Fig. 5. Geometry of the bistatic tandem configuration.

Range time

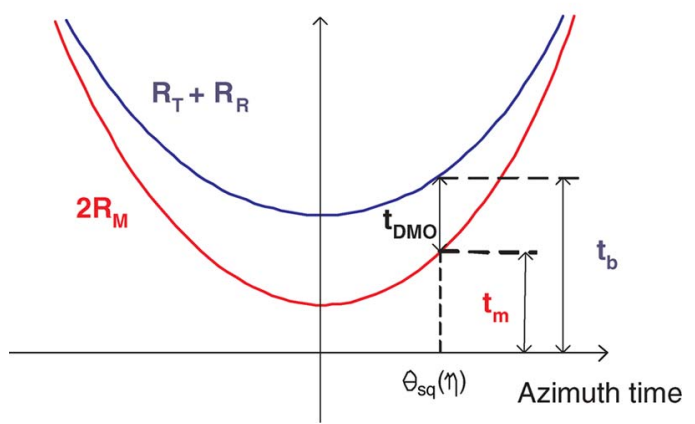

Fig. 6. Squint-dependent travel time of the bistatic and equivalent monostatic configurations. 
In the Appendix, it is shown that the travel times are related by

$$
\begin{aligned}
t_{b}^{2}\left(\theta_{\mathrm{sq}}\right) & \approx t_{m}^{2}\left(\theta_{\mathrm{sq}}\right)+\frac{4 h^{2}}{c^{2}} \cos ^{2} \theta_{\mathrm{sq}} \\
t_{\mathrm{DMO}}\left(\theta_{\mathrm{sq}}\right) & \approx \frac{2 h^{2} \cos ^{2} \theta_{\mathrm{sq}}}{c^{2} t_{b}} .
\end{aligned}
$$

The bistatic platforms are at a constant offset of $2 h$ from each other, and $\theta_{\mathrm{sq}}$ is the squint angle of the equivalent monostatic configuration.

The derivations given in [7] show that the bistatic configuration can be transformed to the monostatic configuration by applying small negative delays $t_{\mathrm{DMO}}$ 's as a function of monostatic squint $\theta_{\mathrm{sq}}$. Applying these negative delays is akin to convolving the bistatic data with the smile operator (the "negative delays" decrease $t_{b}$ to $t_{m}$ ). It was shown in [7] that the smile operator in the 2-D frequency domain for the constant offset case is

$$
\begin{aligned}
H\left(f_{\tau}, f_{\eta}\right) & \approx \exp \left\{j\left(2 \pi\left(f_{o}+f_{\tau}\right)\right) t_{b}\left[1-\sqrt{1-\frac{4 h^{2} \cos ^{2} \theta_{\mathrm{sq}}}{t_{b}^{2} c^{2}}}\right]\right\} \\
& \approx \exp \left\{j\left(2 \pi\left(f_{o}+f_{\tau}\right)\right)\left[\frac{2 h^{2} \cos ^{2} \theta_{\mathrm{sq}}}{t_{b} c^{2}}\right]\right\} \\
& \approx \exp \left\{j\left(2 \pi\left(f_{o}+f_{\tau}\right)\right) t_{\mathrm{DMO}}\left(\theta_{\mathrm{sq}}\right)\right\}
\end{aligned}
$$

where

$$
\cos ^{2} \theta_{\mathrm{sq}}=1-\frac{f_{\eta}^{2} c^{2}}{4 V_{r}^{2}\left(f_{o}+f_{\tau}\right)^{2}}
$$

The geometric relation in (29) is also derived in [1]. These equations are given in [7] but are expressed here in the notations defined in Section II.

Natroshvili and Loffeld [18] showed that Rocca's smile operator becomes the LBF bistatic deformation term by using two approximations

$$
\begin{aligned}
& t_{b} \approx \frac{2 R_{o}}{c} \\
& F^{\frac{3}{2}}=F F^{\frac{1}{2}} \approx F\left(f_{\tau}+f_{o}\right)
\end{aligned}
$$

where

$$
F=\left(f_{\tau}+f_{o}\right)^{2}-\frac{f_{n}^{2} c^{2}}{4 V_{r}^{2}}
$$

and $R_{O}$ is the common range of the closest approach for both the transmitter and receiver. Although not said in [18], it can be shown that the approximation in (31) is equivalent to assuming that $\cos ^{2} \theta_{\text {sq }}$ is approximately equal to $\cos ^{3} \theta_{\text {sq }}$.

By substituting (29)-(31) into (28) and after some algebraic manipulation, the smile operator can be written as

$$
H_{a}\left(f_{\tau}, f_{\eta}\right) \approx \exp \left\{j \phi_{a}\left(f_{\tau}, f_{\eta}\right)\right\}
$$

where

$$
\begin{aligned}
\phi_{a}\left(f_{\tau}, f_{\eta}\right) & =2 \pi\left(f_{\tau}+f_{o}\right) \frac{h^{2} \cos ^{3} \theta_{\mathrm{sq}}}{R_{o} c} \\
& =\frac{2 \pi\left(f_{\tau}+f_{o}\right) h^{2}}{R_{o} c}\left[1-\frac{f_{n}^{2} c^{2}}{4 V_{r}^{2}\left(f_{\tau}+f_{o}\right)^{2}}\right]^{\frac{3}{2}} .
\end{aligned}
$$

In [11], it was shown that, for the constant offset case, the LBF bistatic deformation term in (14) can be expressed by

$\Psi_{2}\left(f_{\tau}, f_{\eta}\right) \approx \frac{2 \pi\left(f_{\tau}+f_{o}\right) h^{2}}{R_{o} c}\left[1-\frac{f_{n}^{2} c^{2}}{4 V_{r}^{2}\left(f_{\tau}+f_{o}\right)^{2}}\right]^{\frac{3}{2}}$.

Thus, smile operator (28) is equal to the bistatic deformation term for the constant offset, tandem case (34), with the approximations (30) and (31).

To arrive at (35), we find that the approximation in (31) is not necessary. Instead of (30), we can use

$$
t_{b} \approx \frac{2 R_{o}}{c \cos \theta_{\mathrm{sq}}}
$$

Geometrically, approximation (36) estimates the slant ranges from the transmitter and receiver to the point target by twice the slant range from the equivalent monostatic platform in the middle of the baseline, i.e., $t_{b} \approx 2 R_{m} / c$. This approximation is adequate when the baseline is small compared to the bistatic range, $2 h / R_{o} \ll 1 / \cos \theta_{\text {sq }}$.

Note that approximation (36) gives a more direct, but equivalent, way of deriving phase (35) than using the two approximations (30) and (31). This is because the $\cos \theta_{\mathrm{sq}}$ term that is missing in (30) is recovered in (31), which uses the approximation $\cos ^{2} \theta_{\text {sq }} \approx \cos ^{3} \theta_{\text {sq }}$.

In essence, Rocca's smile operator can be viewed as a type of bistatic deformation term used in the LBF. Therefore, Rocca's smile operator can be paired with the quasi-monostatic term from the LBF to formulate an alternative point target spectrum.

\section{B. Geometrical Proof of Rocca's Smile Operator}

As seen from the geometry of Figs. 5 and 6, we can represent the bistatic and monostatic travel times by

$$
t_{b}\left(\theta_{\mathrm{sq}}\right)=\frac{R_{o}}{c \cos \theta_{\mathrm{sq} \mathrm{T}}}+\frac{R_{o}}{c \cos \theta_{\mathrm{sqR}}}
$$

$$
t_{m}\left(\theta_{\mathrm{sq}}\right)=\frac{2 R_{o}}{c \cos \theta_{\mathrm{sq}}} .
$$

Applying the cosine rule to the triangles in Fig. 5, we have

$$
\frac{R_{o}}{\cos \theta_{\mathrm{sq}}}=\frac{R_{o}}{\cos \theta_{\mathrm{sq}}}\left[1+\frac{h \cos ^{2} \theta_{\mathrm{sq}}}{R_{o}^{2}}\left(h+\frac{2 R_{o} \sin \theta_{\mathrm{sq}}}{\cos \theta_{\mathrm{sq}}}\right)\right]^{\frac{1}{2}}
$$

$$
\frac{R_{o}}{\cos \theta_{\mathrm{sqR}}}=\frac{R_{o}}{\cos \theta_{\mathrm{sq}}}\left[1+\frac{h \cos ^{2} \theta_{\mathrm{sq}}}{R_{o}^{2}}\left(h-\frac{2 R_{o} \sin \theta_{\mathrm{sq}}}{\cos \theta_{\mathrm{sq}}}\right)\right]^{\frac{1}{2}} .
$$


Performing a binomial expansion on (39) and (40) up to the second-order term and substituting the results into (37), we have

$$
\begin{aligned}
t_{b}\left(\theta_{\mathrm{sq}}\right) & \approx \frac{2 R_{o}}{c \cos \theta_{\mathrm{sq}}}+\frac{h^{2} \cos ^{3} \theta_{\mathrm{sq}}}{c R_{o}}-\frac{h^{4} \cos ^{3} \theta_{\mathrm{sq}}}{4 c R_{o}^{3}} \\
t_{\mathrm{DMO}}\left(\theta_{\mathrm{sq}}\right) & \approx \frac{h^{2} \cos ^{3} \theta_{\mathrm{sq}}}{c R_{o}}-\frac{h^{4} \cos ^{3} \theta_{\mathrm{sq}}}{4 c R_{o}^{3}} .
\end{aligned}
$$

The last term in (42) can be ignored if the baseline is small compared to the bistatic range, $2 h / R_{o} \ll 4$. In a typical satellite case with an $R_{o}$ of $600 \mathrm{~km}$ and a baseline of $10 \mathrm{~km}$, the ratio $2 h / R_{o}$ is 0.017 , and the phase component of the higher order term in (42) has the small value of

$$
\delta \phi=2 \pi f_{o} \frac{h^{4}}{4 R_{o}^{3} c}=0.006 \pi .
$$

Thus, the smile operator becomes

$$
H\left(f_{\tau}, \theta_{\mathrm{sq}}\right) \approx \exp \left\{j 2 \pi\left(f_{\tau}+f_{o}\right) \frac{h^{2} \cos ^{3} \theta_{\mathrm{sq}}}{R_{o} c}\right\} .
$$

It should also be noted that $t_{\mathrm{DMO}}\left(\theta_{\mathrm{sq}}\right)$ in (42) is more accurate for a bistatic SAR configuration as compared to the delay time used in (28), as is evident from the discussion in the Appendix. The time $t_{\mathrm{DMO}}\left(\theta_{\mathrm{sq}}\right)$ in (28) is accurate when it is used to transform a bistatic survey to a monostatic survey in seismic image reconstruction. As the ratio of the baseline to bistatic range becomes small, the signal travel times of (37) and (38) converge.

\section{Simulations-PART 2 (LBF, DMO, AND MSR)}

In this section, we simulate four cases to compare the accuracy of the point target focused using Rocca's smile operator (DMO), the LBF, and the point target spectrum using the MSR.

\section{A. Simulation Parameters}

Four cases of a point target are simulated using the airborne tandem formation parameters given in Table II. The data are focused using the three different spectra. Both platforms operate in stripmap mode, traveling with equal and constant velocity along the same track (the stationary case).

\section{B. Simulation Results}

Again, rectangular weighting is used for both azimuth and range processing. The ideal IRW is 1.06 samples in both range and azimuth. The ideal PSLR is $-13.3 \mathrm{~dB}$, and the ideal ISLR is $-10.0 \mathrm{~dB}$.

1) Case IV: Low Baseline-to-Range Ratio With $\theta_{\mathrm{sqT}}=$ $-\theta_{\mathrm{sqR}}$ : For simulation Case IV, the ratio $2 h / R_{o}$ is small $(0.05)$, and all the point target spectra give accurate processing. The IRW in both range and azimuth differ from the theoretical values by less than $1 \%$. The PSLR and ISLR differ from the theoretical values by less than $1 \mathrm{~dB}$.

2) Case V: Moderate Baseline-to-Range Ratio With $\theta_{\mathrm{sqT}}=$ $-\theta_{\mathrm{sqR}}$ : For simulation Case $\mathrm{V}$, the ratio $2 h / R_{o}$ is 0.124 . The

\begin{tabular}{|c|c|c|}
\hline Simulation parameters & Transmitter & Receiver \\
\hline Platform velocity & $100 \mathrm{~m} / \mathrm{sec}$ & $100 \mathrm{~m} / \mathrm{sec}$ \\
\hline center frequency & \multicolumn{2}{|c|}{$10.17 \mathrm{GHz}$} \\
\hline Range bandwidth & \multicolumn{2}{|c|}{$75 \mathrm{MHz}$} \\
\hline Doppler bandwidth & \multicolumn{2}{|c|}{$232 \mathrm{~Hz}$} \\
\hline Altitude & $1000 \mathrm{~m}$ & $1000 \mathrm{~m}$ \\
\hline \multicolumn{3}{|c|}{ Case IV } \\
\hline Integration time & \multicolumn{2}{|c|}{$6.86 \mathrm{sec}$} \\
\hline Distance between airplanes & \multicolumn{2}{|c|}{$1000 \mathrm{~m}$} \\
\hline Range to point target & $20031 \mathrm{~m}$ & $20031 \mathrm{~m}$ \\
\hline Ratio of baseline to $R_{o}$ & \multicolumn{2}{|c|}{0.05} \\
\hline Squint angle & $-1.43^{\circ}$ & $1.43^{\circ}$ \\
\hline \multicolumn{3}{|c|}{ Case V } \\
\hline Integration time & \multicolumn{2}{|c|}{$2.77 \mathrm{sec}$} \\
\hline Distance between airplanes & \multicolumn{2}{|c|}{$1000 \mathrm{~m}$} \\
\hline Range to point target & $8071 \mathrm{~m}$ & $8071 \mathrm{~m}$ \\
\hline Ratio of baseline to $R_{o}$ & \multicolumn{2}{|c|}{0.124} \\
\hline Squint angle & $-3.55^{\circ}$ & $3.55^{\circ}$ \\
\hline \multicolumn{3}{|c|}{ Case VI } \\
\hline Integration time & \multicolumn{2}{|c|}{$1.80 \mathrm{sec}$} \\
\hline Distance between airplanes & \multicolumn{2}{|c|}{$3000 \mathrm{~m}$} \\
\hline Range to point target & $4026 \mathrm{~m}$ & $4026 \mathrm{~m}$ \\
\hline Ratio of baseline to $R_{o}$ & \multicolumn{2}{|c|}{0.83} \\
\hline Squint angle & $-21.87^{\circ}$ & $21.87^{\circ}$ \\
\hline \multicolumn{3}{|c|}{ Case VII } \\
\hline Integration time & \multicolumn{2}{|c|}{$2.71 \mathrm{sec}$} \\
\hline Distance between airplanes & \multicolumn{2}{|c|}{$1000 \mathrm{~m}$} \\
\hline Range to point target & $5813 \mathrm{~m}$ & $4009 \mathrm{~m}$ \\
\hline Ratio of baseline to $R_{o}$ & \multicolumn{2}{|c|}{0.27} \\
\hline Squint angle & $21.24^{\circ}$ & $50.0^{\circ}$ \\
\hline
\end{tabular}
spectrum obtained using Rocca's smile operator suffers some
TABLE II

Simulation PARAMETERS FOR PART 2-TANDEM CONFIGURATION

phase degradation, leading to a loss of resolution and higher sidelobes (see Fig. 7). The LBF and MSR spectra still provide accurate processing.

3) Case VI: Large Baseline-to-Range Ratio With $\theta_{\mathrm{sqT}}=$ $-\theta_{\mathrm{sqR}}$ : For simulation Case VI, the baseline is increased from 1 to $3 \mathrm{~km}$ to create a large baseline-to-range ratio $2 h / R_{o}=$ 0.83 . Fig. 8 shows that Rocca's smile operator method is not able to focus the point target with this large baseline. In addition, Fig. 9 shows that the focusing limits of the LBF are also reached at this baseline. Fig. 10 shows that the MSR is still able to focus this symmetrical large baseline data correctly by expanding $\widetilde{\eta}_{b}$ in (4) up to the fourth-order azimuth frequency term.

4) Case VII: Moderate Baseline-to-Range Ratio With $\theta_{\mathrm{sqT}} \neq-\theta_{\mathrm{sqR}}$ : The bistatic configurations in Cases IV-VI satisfy condition (24) because $\theta_{\mathrm{sqT}}=-\theta_{\mathrm{sqR}}$. In these symmetrical cases, the LBF is able to maintain accuracy up to large baseline-to-bistatic-range ratios before starting to show phase degradation. This is illustrated in Case VI, which has a very 

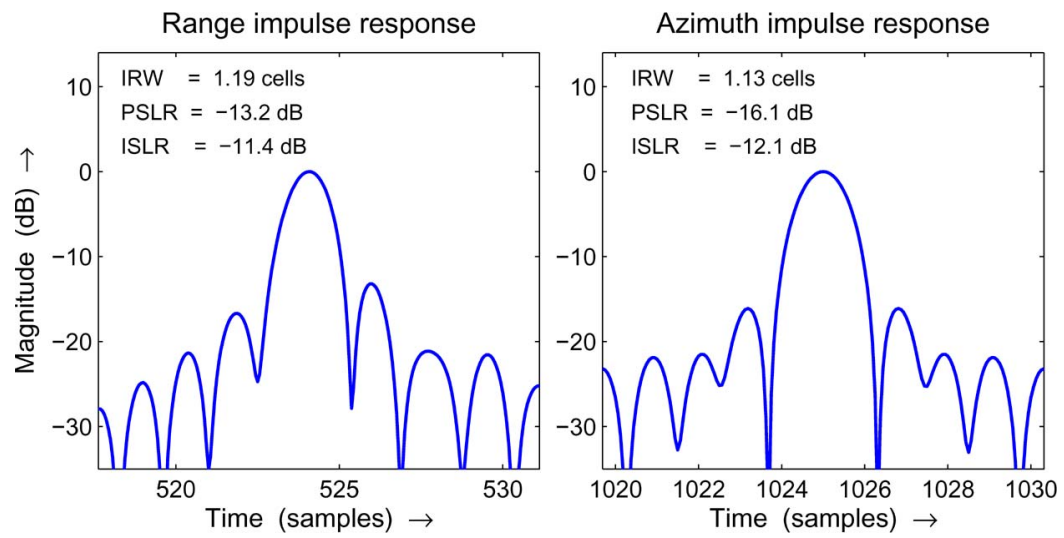

Fig. 7. Point target response focused using Rocca's smile operator.
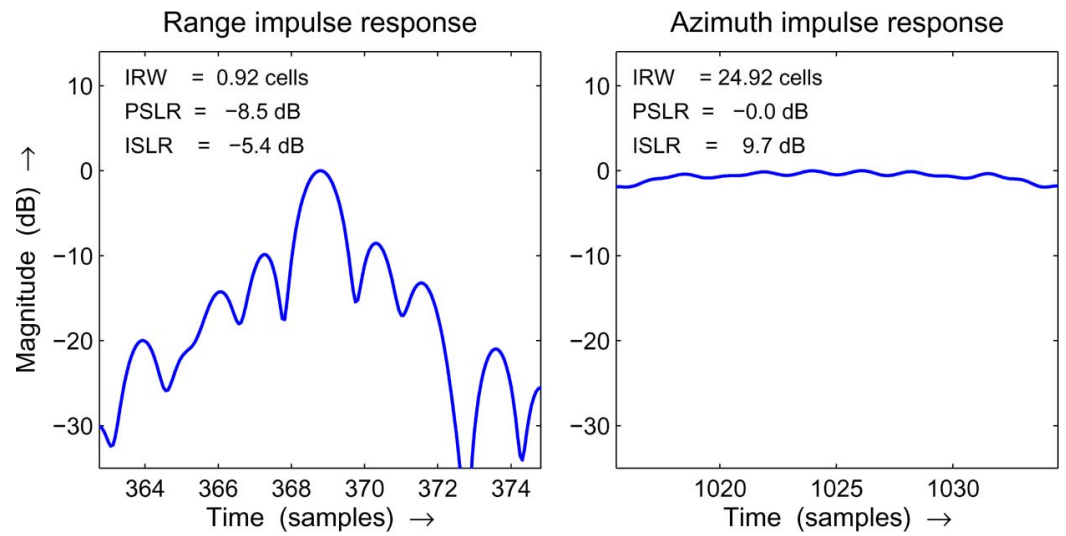

Fig. 8. Point target response focused using Rocca's smile operator.
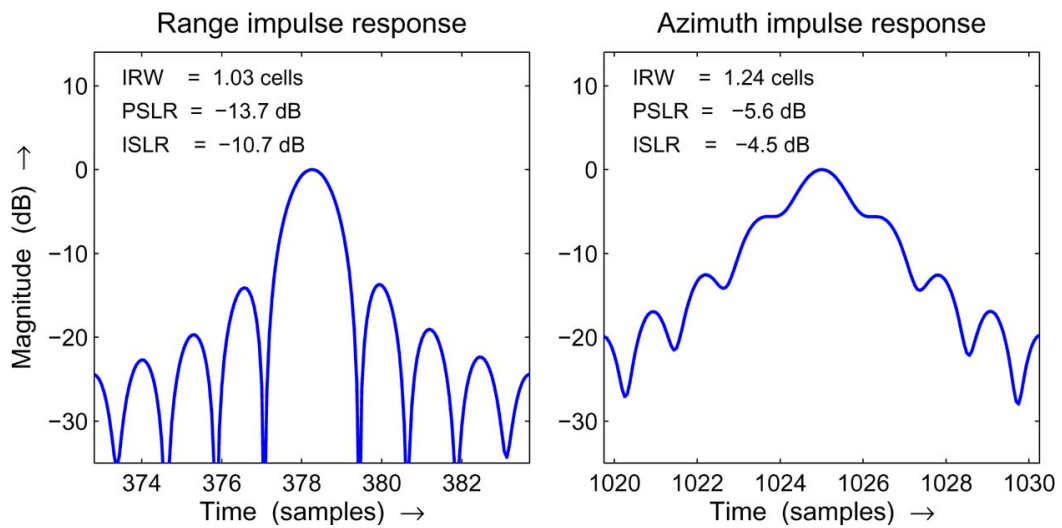

Fig. 9. Point target response focused using the LBF spectrum.

high baseline-to-bistatic-range ratio. Basically, the LBF breaks down only at extreme baseline ratios when $\theta_{\mathrm{sqT}}=-\theta_{\mathrm{sqR}}$.

However, for simulation Case VII, the range vectors are no longer symmetrical, and condition (24) is no longer valid. Even with a shorter baseline and smaller baseline-to-bistatic-range ratio of 0.27 , the point target response in Fig. 11 is worse than the symmetrical Case VI (compare with Fig. 9, where the baseline ratio is 0.83 ).

Fig. 12 shows the impulse response of the point target focused using Rocca's smile operator. For this baseline ratio, the preprocessing method using Rocca's smile operator is not able to focus the point target accurately.
Fig. 13 shows the results, with the MSR spectrum being expanded up to the fourth azimuth frequency term. The accuracy is hardly affected by the change in bistatic configuration (compare with Fig. 10).

\section{Discussion}

The results of this section show that care must be taken to compute the spectrum of the bistatic signal accurately when the signal acquisition geometry departs more and more from the monostatic configuration. The results show that the MSR can retain accuracy better than previous methods under "extreme" 

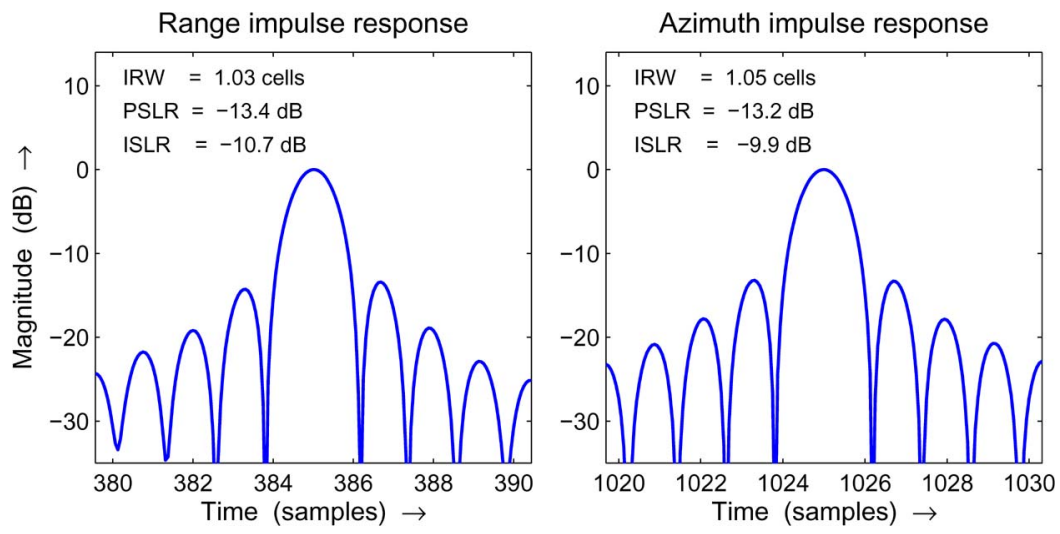

Fig. 10. Point target response focused using the MSR spectrum.
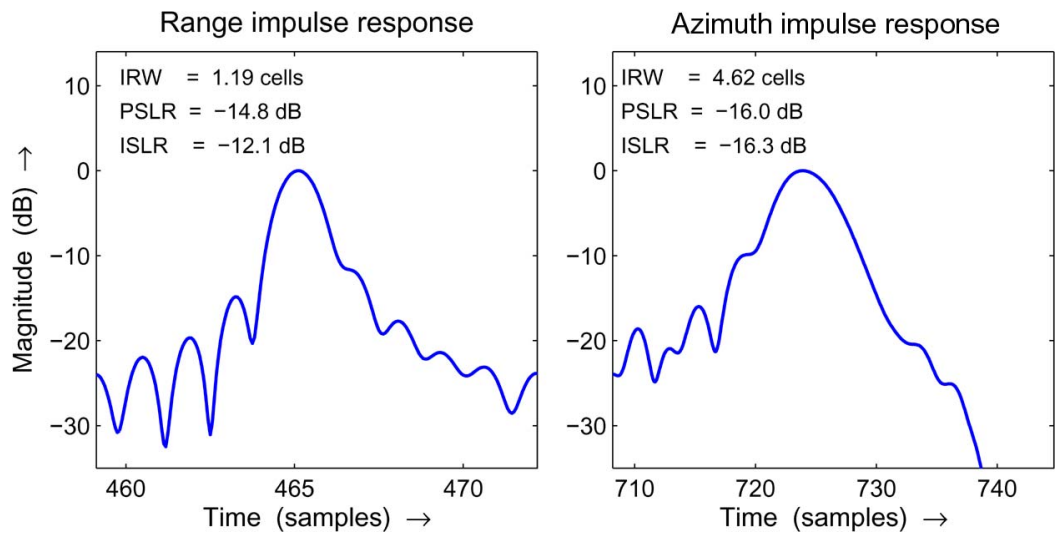

Fig. 11. Point target response focused using Rocca's smile operator.
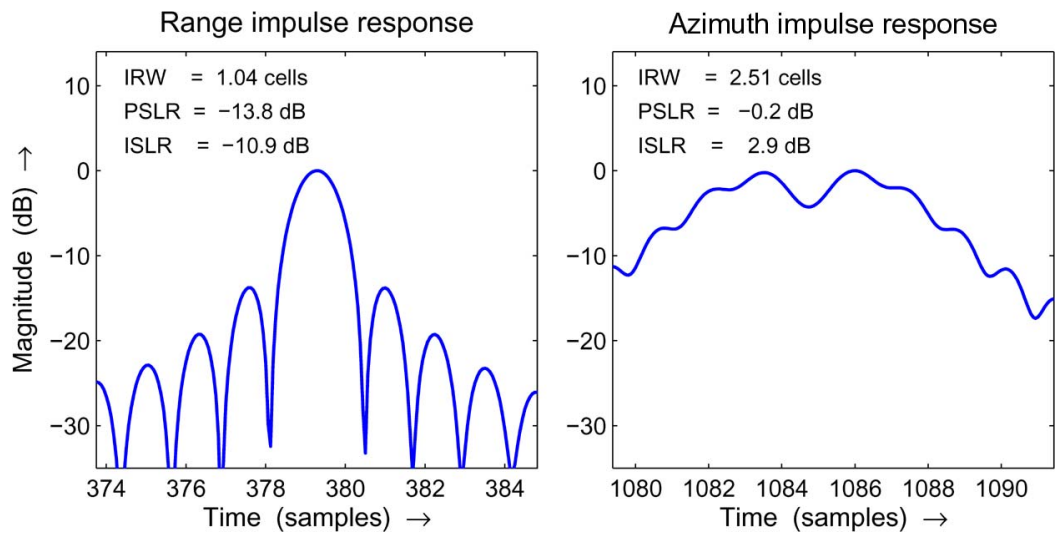

Fig. 12. Point target response focused using the LBF spectrum.
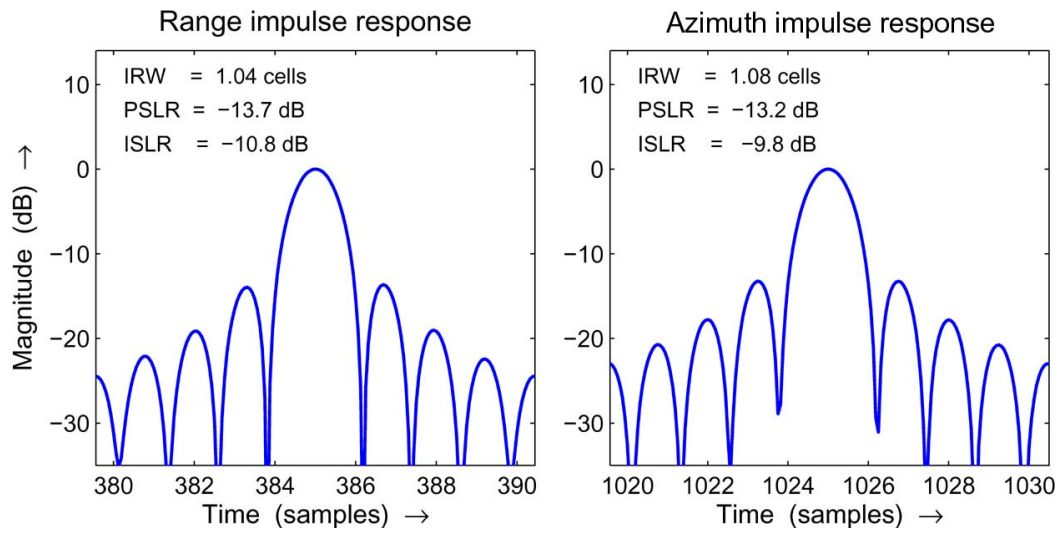

Fig. 13. Point target response focused using the MSR spectrum. 
bistatic configurations with fixed baseline. The MSR can be accurate with nonfixed baselines, as demonstrated by an example in [15].

\section{CONCLUSION}

In this paper, it is shown that, under certain conditions, the MSR, the LBF, and Rocca's smile operator give equivalent representations of the bistatic spectrum. In such cases, all three methods give accurate focusing. Also, it is shown that, under more general conditions, the MSR gives the most accurate representation of the signal spectrum, as the bistatic configuration departs more from the monostatic configuration.

By expanding about the two individual monostatic stationary phase points, it is shown that the LBF is equivalent to a special case of the MSR; the expansion is called the TSPP method. The equivalence occurs when the MSR expansion of the phase terms in the frequency domain is taken only up to the quadratic phase term.

Such an expansion results in a quasi-monostatic term and a bistatic deformation term. The TSPP approach can be considered to be an extension of the LBF because more expansion terms can be used. Bistatic configurations that differ substantially from the monostatic equivalent would require the inclusion of extra expansion terms in the bistatic deformation component. Unfortunately, the use of more terms makes the TSPP approach inefficient.

One way of determining the validity of the LBF is to examine the magnitude of the third- and higher order terms of the TSPP. If they are small, the LBF should provide accurate processing, as the estimated stationary phase point is close to the actual stationary point.

An alternate geometrical method of deriving Rocca's smile operator is shown for the tandem configuration. Using this result, we were able to show a link between Rocca's smile operator (DMO) and the bistatic deformation term of the LBF for the constant offset tandem configuration. It was also found that the accuracy of the DMO method is dependent on the ratio of baseline to bistatic range. A small baseline ratio is required to achieve good accuracy.

To summarize, the point target spectrum of the MSR is the most accurate analytical spectrum among the three spectra. Sufficient terms in the series reversion expansion are required to achieve the desired result. Therefore, if the bistatic degree is high, rather than using the split phase methods such as Rocca's smile, the LBF, or the TSPP, it is recommended to use the bistatic spectrum derived using the MSR.

\section{APPENDIX \\ GEOMETRIC INTERPRETATION OF DMO}

The DMO method, introduced in [7], transforms bistatic acquisitions into monostatic ones for some bistatic configurations, using a convolution operator known as Rocca's smile. This processing technique is a well-known method in the field of seismic reconstruction [9].

Consider the bistatic survey shown in Fig. 14. It consists of a source (transmitter) at point $S$ that sends a seismic pulse, a geophone (receiver) at point $R$, and a dipping or sloping layer

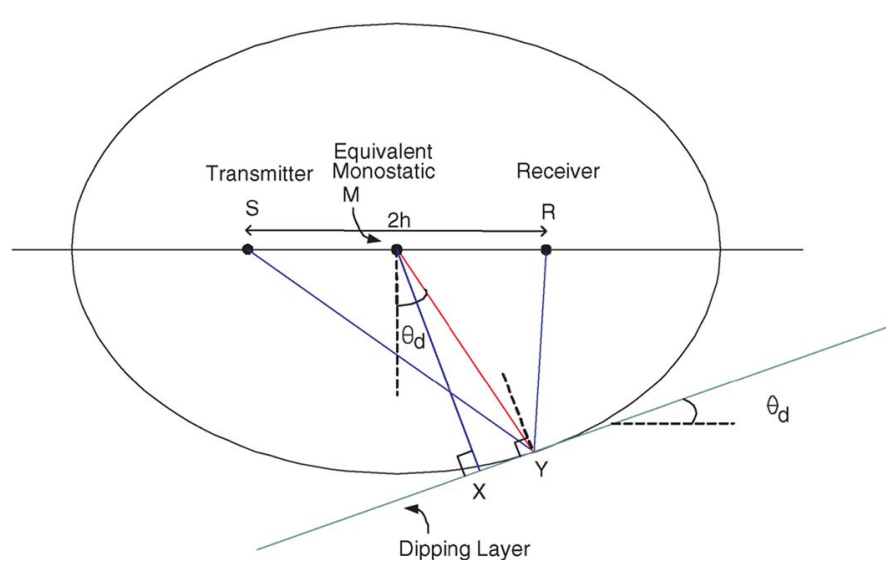

Fig. 14. Bistatic system geometry for computing the DMO operator.

represented by plane $X Y$. The locus of constant delay is an ellipse for this bistatic survey. Using the operator, the bistatic survey can be transformed into a monostatic one, which has a circular locus. Once this can be done, a seismic image can be processed using efficient seismic algorithms [7], [19].

The total time required for the pulse to go from the transmitter to the dipping layer back to the geophone receiver is $t_{b}\left(\theta_{d}\right)$, and the travel path is given by line $S Y$ plus line $Y R$. For an equivalent monostatic case, the source and receiver are located at the middle of the baseline; the time required is given by $t_{m}\left(\theta_{d}\right)$, with the travel path being given by line $M X$ plus line $X M$.

Using the geometry shown in Fig. 14, it can be shown that the bistatic and monostatic time delays are related by

$$
t_{b}^{2}\left(\theta_{d}\right)=t_{m}^{2}\left(\theta_{d}\right)+\frac{4 h^{2}}{c_{e}^{2}} \cos ^{2} \theta_{d}
$$

where $c_{e}$ is the speed of the pulse in the homogenous medium.

The bistatic signal has a longer time delay than the monostatic signal, i.e., $t_{b}\left(\theta_{d}\right)>t_{m}\left(\theta_{d}\right)$. The small difference in signal arrival time or "negative delay" between $t_{b}\left(\theta_{d}\right)$ and $t_{m}\left(\theta_{d}\right)$ is known as the DMO in seismic terminology

$$
t_{\mathrm{DMO}}\left(\theta_{d}\right)=t_{b}\left\{1-\sqrt{1-\frac{4 h^{2} \cos ^{2} \theta_{d}}{t_{b}^{2} c_{e}^{2}}}\right\} .
$$

Thus, by applying different negative delays as a function of the dipping angle, the bistatic survey can be transformed to a monostatic survey.

To transform the seismic representation to a bistatic SAR model, the dipping layer is removed from the diagram and replaced with a point target at position $Y$. The bistatic survey is similar to the bistatic SAR system with a fixed baseline of $2 h$, with a transmitter at point $S$, and a receiver at point $R$. While the bistatic path is the same for both the seismic and radar cases, the monostatic path is given by line $M Y$ plus line $Y M$. If the baseline is short compared to the bistatic range, the lengths of $M Y$ and $M X$ will be almost the same. Thus, we can make the approximation given in (26), with $c_{e}$ of (46) being replaced by $c$ and $\theta_{d}$ by the squint angle $\theta_{\mathrm{sq}}$ of the equivalent monostatic SAR system. 


\section{ACKNOWLEDGMENT}

The authors would like to thank Prof. O. Loffeld from the Center for Sensorsystems (ZESS), University of Siegen, Siegen, Germany, for hosting Dr. Y. L. Neo as a Visiting Scientist in 2006 and for the useful discussions on Rocca's smile. The authors would also like to thank the reviewers, whose comments led to substantial improvements in this paper.

\section{REFERENCES}

[1] I. G. Cumming and F. H. Wong, Digital Processing of Synthetic Aperture Radar Data: Algorithms and Implementation. Norwood, MA: Artech House, 2005.

[2] A. Papoulis, Signal Analysis. New York: McGraw-Hill, 1977.

[3] I. Walterscheid, J. H. G. Ender, A. R. Brenner, and O. Loffeld, "Bistatic SAR processing and experiments," IEEE Trans. Geosci. Remote Sens., vol. 44, no. 10, pp. 2710-2717, Oct. 2006.

[4] V. Giroux, H. Cantalloube, and F. Daout, "An Omega-K algorithm for SAR bistatic systems," in Proc. IGARSS, Seoul, Korea, Jul. 2005, pp. 1060-1063.

[5] M. Soumekh, "Bistatic synthetic aperture radar inversion with application in dynamic object imaging," IEEE Trans. Signal Process., vol. 39, no. 9, pp. 2044-2055, Sep. 1991.

[6] R. Bamler, F. Meyer, and W. Liebhart, "Processing of bistatic SAR data from quasi-stationary configurations," IEEE Trans. Geosci. Remote Sens., vol. 45, no. 11, pp. 3350-3358, Nov. 2007.

[7] D. D'Aria, A. Monti Guarnieri, and F. Rocca, "Focusing bistatic synthetic aperture radar using dip move out," IEEE Trans. Geosci. Remote Sens., vol. 42, no. 7, pp. 1362-1376, Jul. 2004.

[8] A. Monti Guarnieri and F. Rocca, "Reduction to monostatic focusing of bistatic or motion uncompensated SAR surveys," Proc. Inst. Electr. Eng.-Radar, Sonar Navig., vol. 153, no. 3, pp. 199-207, Jun. 2006.

[9] D. Hale, "Dip-moveout by Fourier transform," Geophysics, vol. 49, no. 14, pp. 741-757, Jun. 1984.

[10] R. Bamler and E. Boerner, "On the use of numerically computed transfer function for processing of data from bistatic SARs and high squint orbital SARs," in Proc. IGARSS, Seoul, Korea, 2005, vol. 2, pp. 1051-1055.

[11] O. Loffeld, H. Nies, V. Peters, and S. Knedlik, "Models and useful relations for bistatic SAR processing," IEEE Trans. Geosci. Remote Sens., vol. 42, no. 10, pp. 2031-2038, Oct. 2004.

[12] O. Loffeld, H. Nies, H. Gebhardt, V. Peters, and S. Knedlik, "Bistatic SAR-Some reflections on Rocca's smile," in Proc. EUSAR, Ulm, Germany, May 2004, pp. 379-383.

[13] K. Natroshvili, O. Loffeld, H. Nies, A. M. Ortiz, and S. Knedlik, "Focusing of general bistatic SAR configuration data with 2-D inverse scaled FFT," IEEE Trans. Geosci. Remote Sens., vol. 44, no. 10, pp. 2718-2727, Oct. 2006.

[14] M. Rodríguez-Cassolá, G. Krieger, and M. Wendler, "Azimuth-invariant, bistatic airborne SAR processing strategies based on monostatic algorithms," in Proc. IGARSS, Seoul, Korea, Aug. 2005, pp. 1047-1050.

[15] Y. Neo, F. Wong, and I. G. Cumming, "A two-dimensional spectrum for bistatic SAR processing using series reversion," IEEE Geosci. Remote Sens. Lett., vol. 4, no. 1, pp. 93-96, Jan. 2007.

[16] P. M. Morse and H. Feshbach, Methods of Theoretical Physics, 1st ed. New York: McGraw-Hill, 1953.

[17] R. K. Raney, "A new and fundamental Fourier transform pair," in Proc. IGARSS, Clear Lake, TX, May 1992, pp. 106-107.

[18] K. Natroshvili and O. Loffeld, "Comparison of bistatic SAR focusing approaches," in Proc. EUSAR, Dresden, Germany, May 16-18, 2006. CD-ROM

[19] R. H. Stolt, "Migration by transform," Geophysics, vol. 43, no. 1, pp. 2348, Feb. 1978.

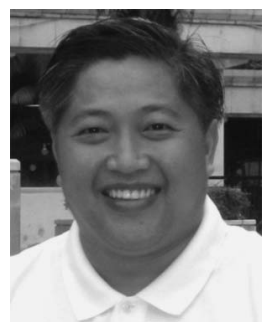

Yew Lam Neo received the B.Eng. degree in electrical engineering from the National University of Singapore, Singapore, in 1994, and the Ph.D. degree from the University of British Columbia, Vancouver, BC, Canada, in 2007.

From 1994 to 2001, he was an Engineer with DSO National Laboratories, Singapore, where he worked on SAR systems and software for real-time embedded systems. In 2006, he was a Visiting Scientist for three months with the Center for Sensorsystems (ZESS), University of Siegen, Siegen, Germany. He is currently with the Sensor Division, Radar Techniques Laboratory, DSO National Laboratories, where he is continuing his research in bistatic SAR signal processing.

Dr. Neo was awarded the Engineering Book Prize and was the recipient of the Innovation Prize Award for his final year project work on a very high frequency synthesizer.

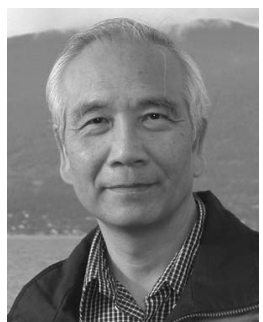

Frank H. Wong received the B.Eng. degree in electrical engineering from McGill University, Montreal, QC, Canada, the M.Sc. degree in electrical engineering from Queen's University, Kingston, ON, Canada, and the $\mathrm{Ph} . \mathrm{D}$. degree in computer science from the University of British Columbia (UBC), Vancouver, BC, Canada.

Since 1977, he has been with MacDonald, Dettwiler and Associates Ltd., Richmond, BC, Canada, where he worked in the Landsat and SPOT imaging area for the first few years. Then, his interest switched to SAR, and he has been working on airborne and satellite SAR processing and Doppler estimation ever since. He is also with the Radar Remote Sensing Group, Department of Electrical Engineering, UBC, where he has been a Sessional Lecturer on image processing for 18 years. He was a Visitor to the National University of Singapore, Singapore, for a year in 1999, where he did research in bistatic SAR processing.

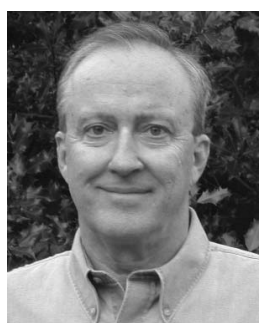

Ian G. Cumming (S'63-M'66-SM'05-LSM'06) received the B.Sc. degree in engineering physics from the University of Toronto, Toronto, ON, Canada, in 1961, and the Ph.D. degree in computing and automation from Imperial College, University of London, London, U.K., in 1968.

In 1977, he was with MacDonald, Dettwiler and Associates Ltd., Richmond, BC, Canada, where he developed SAR signal processing algorithms, including Doppler estimation and autofocus routines. $\mathrm{He}$ has been involved in the algorithm design of digital SAR processors for SEASAT, SIR-B, ERS-1/2, J-ERS-1, and RADARSAT, as well as several airborne radar systems. He was a Visiting Scientist with the German Aerospace Center (DLR), Oberpfaffenhofen, for one year in 1999. Since 1993, he has been with the Department of Electrical and Computer Engineering, University of British Columbia, Vancouver, BC, Canada, where he is the MacDonald Dettwiler/NSERC Industrial Research Chair in radar remote sensing. The Radar Remote Sensing Laboratory has published papers in the fields of SAR processing, SAR data encoding, satellite SAR two-pass interferometry, airborne along-track interferometry, polarimetric radar image classification, and SAR Doppler estimation. 